\title{
Surgery was successful - but how did it go for the patient? Experiences from and hopes for the Swedish Perioperative Register
}

Michelle Chew

The self-archived postprint version of this journal article is available at Linköping University Institutional Repository (DiVA):

http:/ / urn.kb.se/ resolve?urn=urn:nbn:se:liu:diva- 145332

N.B.: When citing this work, cite the original publication.

Chew, M., Mangelus, C., Enlund G., Spetz, P., and Lyckner, S.,(2015), Surgery was successful - but how did it go for the patient? Experiences from and hopes for the Swedish Perioperative Register, European J ournal of Anaesthesiology, 32(7), 453-454. https:// doi.org/ 10.1097/

EJA.0000000000000283

Original publication available at:

https:// doi.org/ 10.1097/ EJ A.0000000000000283

Copyright: Lippincott, Williams \&Wilkins

http:// www.lww.com/ 


\title{
Surgery was successful - but how did it go for the patient? Experiences from and hopes for the Swedish Perioperative Register
}

\author{
Michelle S. Chew, Claes Mangelus, Gunnar Enlund, Peter Spetz, Sara Lyckner, for the Swedish \\ Perioperative Register
}

European Journal of Anaesthesiology 2015, 32:453-454

The Swedish Perioperative Register (SPOR) was founded in 2011 as a development of the earlier Swedish Anaesthesia Register under the auspices of the Swedish Society of Anaesthesiology and Intensive Care Medicine. In October 2014, it became a nonprofit organisation and Sweden's first official perioperative register. It is funded by participating members, as well as partly through grants from the Swedish Association of Local Authorities and Regions.

The broad aims of the registry are to aid in the follow-up and evaluation of perioperative outcomes on a national basis, both in the short and long term; to act as an instrument for local and regional follow-up and for perioperative improvement initiatives; to act as a basis for scientific research and quality improvement strategies; and to abide by the rules, recommendations and guidelines pertaining to national quality registers.

The registry covers the entire perioperative process. It has been implemented electronically within most Swedish operative units. By the end of 2016, we anticipate that $95 \%$ of surgical procedures will be included. As a first step, a basic module for registering perioperative variables was created. This consists of 82 variables, of which 16 are compulsory. Examples of compulsory variables include type and urgency of surgery, surgical and anaesthetic procedure codes and times, cancellations and the occurrence of untoward perioperative events. A second module has recently been completed, consisting of a postoperative complications list, covering events up to $24 \mathrm{~h}$ in the postanaesthesia recovery unit (PACU). Postoperative events for patients admitted directly to ICUs are not encompassed by the register yet. However, the occurrence of planned and unplanned postsurgical admissions to ICU are registered.

We strive to use national and international definitions and standards where possible, for example, Systematized Nomenclature of Medicine - Clinical Terms (SNOMED-CT), Well Being, Integrity, Prevention and Safety (VIPS) model for nursing documentation. The basic output is presented as predefined reports. At present, there are 11 predefined reports that are available online to registered users, with a further five planned. The reports are available as local data and national data and cover a range of process and quality measures, including cancellations, the use of the WHO surgical checklist, unexpected perioperative events, postoperative pain and nausea/vomiting, short and long-term mortalities.

SPOR recognises the need to involve various parties interested in the perioperative process in order to obtain as comprehensive and accurate data as possible. During its evolution, contacts were established and input sought from representatives of nurse anaesthetists, surgical nurses and surgical specialties. Consideration is given to the wide breadth of surgical procedures, from major inpatient surgery to day surgery, from the elderly to paediatric, from university hospitals to rural hospitals, and from public care to private care. Appropriate Patient Associations will also be contacted.

An estimated 600000 surgical procedures are conducted in Sweden annually (http://www.nysam.com/report/ form). This type of database therefore provides unprecedented opportunities for answering questions regarding a large proportion of the population. The existence of government-administered health registries and personal identification numbers also allow long-term follow-up, 
which is impossible in many other countries. ${ }^{1}$ There are also opportunities for joint processing of data from multiple registers, although significant infrastructural and legal barriers still exist. This was recognised by the Swedish government, and in 2013, the Swedish Research Council was commissioned to investigate the legal impediments for register-based research. The results of this commission have now been published and its proposals are currently being considered by the various interested parties, including the Swedish Society for Anaesthesiology and Intensive Care. ${ }^{2}$

Data protection and patient confidentiality are foremost considerations. Patients are protected by Swedish law (Patient Data Act SFS2008:355). They are informed that their data will be recorded in SPOR and that these data cannot be collected if they actively decide to 'opt out'. Patients are also informed that they can request a complete erasure of their data from the register if they wish. In Sweden, an individual cannot assume legal responsibility for a quality register, so this responsibility is usually held by county councils. For SPOR, the registry centre is the Uppsala Clinical Research Centre, with the Uppsala county council ultimately responsible for data. The chairman of SPOR is the registrar and has the delegated responsibility for data storage and its appropriate usage.

SPOR is a newly established register and we have many aspirations for its future. In its most basic form, we will be able to document numbers, types, durations and urgencies of surgical procedures, cancellations, anaesthesia type, mortality rates and perioperative unexpected events. For perioperative medicine, this represents a huge step forward in quality assurance and clinical governance, providing reliable and basic data upon which to build. In this regard, we wish to emulate the success of other well established registries such as the American College of Surgeons National Surgical Quality Improvement Program that has demonstrated a more than $40 \%$ decrease in mortality and morbidity between 1991 and 2006 (http://site.acsnsqip.org/program-specifics/nsqiphistory/). In Sweden, the success of the SWEDEHEART registry is an example of a well validated registry showing how national audit can contribute to international comparative effectiveness research. ${ }^{3}$

We recognise major limitations with SPOR, such as the inability to follow-up patients admitted to ICU, and complications beyond $24 \mathrm{~h}$ of surgery. Another real threat to the register, as for other registers, is the question of workload. At present, most data are obtained from electronic surgical planning systems, without further manual input. Some variables such as per-operative and postoperative complications and their severity are entered manually. Our principle, however, is to maximise automatic data transfer without the requirement for manual input.

In the future, we plan to use the year 2015 for validation purposes, concentrating on data integrity and completeness. We will continue to work on possibilities for merging national databases, specifically, with other well established surgical registries and the Swedish Intensive Care Register. One important goal in 2015 to 2016 will be the development of a postoperative complications module covering the postsurgical period until hospital discharge.

SPOR is a national perioperative register with wide coverage and encompasses a large number of process and quality variables. Not only are we are learning how best to deal with these huge amounts of data but also we are exploring ways of exploiting other national registries and databases to maximise the appropriate collection of information. We hope to be able to provide a reliable database for national audit, identification of high-risk patients and designing better peri-operative services.

\section{Acknowledgements relating to this article}

Assistance with the editorial: the authors acknowledge efforts of Anders T Larsson, Björn Holmström, Claes Frostell and Sophie Lindgren, as well as staff at the Uppsala Clinical Research Centre in running and maintaining the register. We are also indebted to endusers at Swedish hospitals for contributing data.

Financial support and sponsorship: the Swedish Perioperative Register is funded by participating members and through grants from the Swedish Association of Local Authorities and Regions.

Conflicts of interest: none.

Comment from the editor: this editorial was checked by the editors but was not sent for external peer-review. MSC is an associate editor of the Eur J Anaesthesiol.

\section{References}

1 Emilsson L, Lindahl B, Köster M, et al. Review of 103 Swedish Healthcare Quality Registries. J Int Med 2015; 277:94-136.

2 Swedish Government Official Reports SOU 2014:45. Unique knowledge through registry research. Report of registry research investigation. Elanders Sweden AB. Stockholm. ISBN 978-91-38-24133-2.

3 Chung S-C, Gedeborg R, Nicholas O, et al. Acute myocardial infarction: a comparison of short-term survival in national outcome registries in Sweden and the UK. Lancet 2014; 383:1305-1312. 